\title{
Olfaction after endoscopic sinus surgery: long-term results*
}

\author{
Hans Rudolf Briner ${ }^{1}$, Nick Jones², Daniel Simmen¹ \\ ORL-Zentrum (Center for Otology, Skull Base Surgery, Rhinology and Facial Plastic Surgery), \\ Hirslanden Clinic, Zurich, Switzerland \\ Department of Otorhinolaryngology, Head and Neck Surgery, University Hospital, Nottingham, United Kingdom
}

Rhinology 50: 178-184, 2012

DOI:10.4193/Rhino11.213

*Received for publication:

October 2, 2011

Accepted: January 24,2012

\begin{abstract}
Summary
Loss of olfactory function is one of the main symptoms in patients with chronic rhinosinusitis. This prospective, non-randomized interventional study reports five years results of olfaction of patients with chronic rhinosinusitis who underwent endoscopic sinus surgery in conjunction with topical medical treatment. Forty-five patients with chronic rhinosinusitis who underwent endoscopic sinus surgery were evaluated preoperatively, after three months and 34 (76\%) of them after five years. Olfactory function was assessed by a subjective visual analogue scale, by a screening test of olfaction with Smell Diskettes and by measuring the N-Butanol threshold. Patient's subjective sense of olfaction using a visual analogue scale was improved in $79 \%$ at 5 years. Objective measurements by Smell Diskettes improved in $53 \%$ at 5 years whilst the quantitative measurement by the N-Butanol threshold improved in $85 \%$ at 5 years. The high percentage of patients with improvement of olfaction five years after surgery indicates that endoscopic sinus surgery in conjunction with continued topical treatment leads to a long term improvement of the sense of smell. However, it was found that - measured by the sensitive N-Butanol threshold up to $9 \%$ had no improvement and $6 \%$ had deterioration in their olfaction at 5 years after endoscopic sinus surgery. This fact has to be considered in the preoperative counselling of patients.
\end{abstract}

Key words: olfaction, endoscopic sinus surgery, rhinosinusitis, long-term results

\section{Introduction}

Impairment of the sense of smell is one of the main symptoms of patients with chronic rhinosinusitis ${ }^{(1,2)}$. The loss of olfaction can reduce quality of life and may even interfere with someone's professional capacities such as cooks, firefighters and vintners. The severity of the loss of the sense of smell usually parallels the severity of the underlying chronic rhinosinusitis ${ }^{(3)}$. Successful treatment of patients with chronic rhinosinusitis therefore has the potential to reduce or normalize the loss of olfaction ${ }^{(4)}$. Current concepts in the treatment of chronic rhinosinusitis centre on the use of local and systemic medication ${ }^{(2)}$. If medical treatment fails to control the patient's symptoms, endoscopic sinus surgery is indicated. It is known that endoscopic sinus surgery can improve the sense of smell in patients with loss of olfaction due to chronic rhinosinusitis ${ }^{(5-11)}$. However, in most of the studies the sense of smell has only been evaluated less than one year after surgery. This paper presents a prospective evaluation of long-term results of the olfactory function in patients, which had endoscopic sinus surgery for chronic rhinosinusitis.

\section{Material and methods}

\section{Study design}

The study was designed as a prospective, non-randomized interventional trial. Patients aged eighteen years or more who had chronic rhinosinusitis with or without polyps as defined by their clinical history, physical examination and radiological findings ${ }^{(2)}$, and who were candidates to undergo endoscopic sinus surgery were enrolled in the trial. All patients failed to respond sufficiently to conservative medical treatment such as topical saline douches, topical steroids and short courses of systemic steroids. Patients who had previous sinus surgery were excluded. The severity of the disease was rated according to the changes in the computed tomography (CT) according to the Lund-Mackay grading system $^{(12,13)}$. 


\section{Surgery}

Endoscopic sinus surgery was performed between November 2003 and December 2004 at the ORL-Zentrum, Hirslanden Clinic, Zurich, by the authors (BHR, SD). Depending on the individual severity of the disease, a partial anterior and posterior ethmoidectomy, a sphenoethmoidectomy or a frontosphenoethmoidectomy was performed on both sides under general anesthesia. Special attention was given to achieve an open olfactory cleft at the end of surgery. If there was a blockage of the olfactory cleft despite the ethmoidectomy, a gentle lateralisation of the middle turbinate was performed respecting the anterior superior origin and the posterior inferior origin (inferior part of the ground lamella) of the middle turbinate to avoid a postoperative blockage of the frontoethmoidal and maxilloethmoidal drainage pathways. Postoperatively, the patients were followed up with yearly visits and medical treatment for the chronic rhinosinusitits was titrated with douching and topical nasal steroids according to the symptoms and activity of residual disease. None of the patients received a course of systemic steroids between surgery and the first testing after three months nor within 3 months of the tests done at 5 years. Only one patient with severe chronic rhinosinusitis with polyps (Samter's triad) had a revision frontoethmoidectomy with an additional frontal sinus drill out procedure due to recurrence of the polyps three years after the initial operation. Endoscopic examination was performed after 3 months and after 5 years to rule out scar formation of the olfactory cleft.

\section{Olfactory function assessment}

The sense of smell was assessed before surgery, three months after surgery and five years after surgery. The patient's subjective olfactory function was assessed by a visual analogue scale (VAS) resulting in a value between 0 (no sense of smell at all) and 100 (normal sense of smell). A screening test of olfaction was performed with 'Smell Diskettes' resulting in a score between 0 and $8{ }^{(14)}$. A score of 7 and 8 is regarded as normal; a score of 6 and less indicates hyposmia or anosmia. Additionally, a quantitative test of olfaction was performed by measuring the olfactory threshold of N-Butanol ${ }^{(15)}$. Squeezing bottles of N-Butanol with a dilution series of 9 bottles of $\mathrm{N}$-Butanol between concentrations of $4 \%$ up to a dilution of $0.00061 \%$ were used. The threshold was determined by presenting a bottle without N-Butanol and one out of the dilution series beginning at the highest concentration. The patient had to detect the N-Butanol three times before a next dilution was presented. The bottle with the highest concentration of $\mathrm{N}$-Butanol of $4 \%$ was defined as score 1 , the bottle with the lowest concentration was defined as score 9. If an anosmic patient did not recognize bottle 1 with the highest concentration of N-Butanol, he was 'scored' as 0 . So the N-Butanol threshold measurement resulted in a score between 0 (anosmia) and 9 (maximal sensitivity). Among the three methods of assessment of olfactory function used in this study, this threshold test can be regarded as the most accurate method, reflecting the olfactory performance most precisely.

The study was accepted by the ethics committee of the Canton Zurich, Switzerland.

\section{Statistics}

For statistical analysis mean values with standard deviation as well as median values with interquartile range (IQR) were calculated. Differences between the preoperative and postoperative olfaction test scores were analyzed using the Wilcoxon signed ranks test. $P$ values of $<0.05$ were considered to be statistically significant. However, in situations where the Bonferroni-correction had to be used, a $p$ value of $<0.017$ was regarded as significant. Correlations between the preoperative CT-score and preoperative olfaction scores, and their respective changes in olfaction scores after 3 months and 5 years were evaluated using the Spearman correlation. The same method was used to analyze correlations between the three different olfaction tests (VAS, Smell Diskettes and N-Butanol threshold). Statistical analysis was performed using IBM SPSS Statistics 19 (SPSS Inc., Chicago, IL, USA).

\section{Results}

Forty-five patients ( 30 males, 15 females) with a mean age of 50.2 years ( 24 years -77 years) were enrolled in the study. All of them could be assessed after three months and 34 (76\%) could be evaluated again after five years. According to the Lund and Mackay scoring system, 16 patients (36\%) had limited opacification of the paranasal sinuses (CT scan score 4 - 13 points), and 29 patients (64\%) had extensive mucosal swelling (CT scan score 14 - 24 points). The CT score correlated with the clinical differentiation between the subgroups of chronic rhinosinusitis without nasal polyps and the group with nasal polyps. So for subgroup analysis the 16 patients with a CT score below 14 had no polyps and the 29 other patients had polyps. There were no patients with allergic fungal rhinosinusitis among the patients enrolled for the study.

The subjective assessment of the sense of smell with a visual analog scale $(0$ - 100) resulted in a mean value of 28.4 (range 0 - 100) preoperatively, a mean value of 69.1 (range 1 - 100) after three months and a mean value of 58.5 (range 1 - 100) after five years (Figure 1). The improvement of the mean VAS value compared with the preoperative value was significant for 3 months ( $p<0.001)$ and for 5 years $(p<0.001)$. The tendency to a lower value after 5 years compared with the value 3 months postoperatively did not reach significance $(p=0.037$; level of significance with Bonferroni-correction $<0.017$ ). Analyzing the individual smell performance, 39 of 45 patients (87\%) had 
Table 1. Mean values with standard deviation and median values with the interquartile range (IQR) of the age, the preoperative CT-score and the preand postoperative olfaction scores.

\begin{tabular}{|c|c|c|c|c|}
\hline & $\mathbf{n}$ & Mean & $\begin{array}{l}\text { Standard } \\
\text { deviation }\end{array}$ & Median (IQR) \\
\hline Age & 45 & 50.2 & 13.4 & $53(38.5-60.5)$ \\
\hline Preoperative CT-score & 45 & 14.5 & 5.8 & $14(10.5-20)$ \\
\hline Preoperative Visual Analogue Scale (VAS) & 45 & 28.4 & 32.4 & $14(2-54)$ \\
\hline VAS after 3 months & 45 & 69.1 & 29.7 & $73(57-93)$ \\
\hline VAS after 5 years & 34 & 58.5 & 31.2 & $65.5(28.3-84)$ \\
\hline Preoperative Smell Diskettes score & 45 & 5.1 & 2.6 & $5(3-8)$ \\
\hline Smell Diskettes after 3 months & 45 & 7.1 & 1.5 & $8(7-8)$ \\
\hline Smell Diskettes after 5 years & 34 & 6.1 & 2.2 & $7(5-8)$ \\
\hline Preoperative N-Butanol threshold score & 45 & 2.1 & 2.2 & $2(0-4)$ \\
\hline N-Butanol score after 3 months & 45 & 4.6 & 2.2 & $4(3-6)$ \\
\hline N-Butanol score after 5 years & 34 & 5.1 & 2.1 & $6(4-6)$ \\
\hline
\end{tabular}

a higher postoperative VAS score after three months and 27 of 34 patients (79\%) after five years. One patient (2\%) showed an unchanged performance after three months and also after five years, whereas 5 of 45 patients (11\%) showed a lower score after three months and 6 of 34 patients (18\%) after five years.

The screening test with smell diskettes ( 0 - 8 points) showed a mean score of 5.1 (range 0 - 8) preoperatively, a mean score of 7.1 (range 2 - 8) at three months and a mean score of 6.1 (range 0 - 8) after five years (Figure 2). The improvement of the mean score compared with the preoperative score was significant for 3 months $(p<0.001)$ but did not reach significance after 5 years $(p=0.063)$. On the other hand, the decrease of the score after 5 years compared with the score 3 months postoperatively was significant $(p<0.001)$. Analyzing the individual smell performance, 28 of 45 patients (62\%) had a higher postoperative score after three months and 18 of 34 (53\%) after five years. Twelve patients (27\%) showed an unchanged performance after three months and 3 (9\%) after five years, whereas 5 of 45 patients $(11 \%)$ showed a lower score after three months and 13 of 34 patients (38\%) after five years. The Smell diskettes test defines patients having a score of 7 and 8 as 'normosmics,' patients reaching a score of 6 or less as 'hyposmics.' Using this definition, 17 of 45 patients (38\%) were normosmics before undergoing endoscopic sinus surgery and 35 of 45 patients (78\%) were normosmics after 3 months. After 5 years, 19 of 34 patients (56\%) could be defined as normosmics.

The olfactory threshold measurement using the N-Butanol bottles in a dilution series (score between 0 and 9) resulted in a mean preoperative score of 2.1 (range 0 - 7). After three months, the mean score was 4.6 (range 0 - 9) and after five years the mean score was 5.1 (range 0 - 9) (Figure 3). The improvement of the mean $\mathrm{N}$-Butanol threshold score compared with the preoperative score was significant for 3 months $(p<0.001)$ and for 5 years $(p<0.001)$. The tendency to a higher score after 5 years compared with the score 3 months postoperatively did not reach significance $(p=0.10)$. Analyzing the individual smell performance, 37 of 45 patients (82\%) had a higher score after three months and 29 of 34 (85\%) after five years. Four patients (9\%) showed an unchanged performance after three months and 3 (9\%) after five years, whereas 4 patients (9\%) showed a lower score after three months and 2 (6\%) after five years. One of these patients had chronic rhinosinusitis 'without' polyps (preoperative CT score: 10), whereas the other had severe nasal polyposis (preoperative CT score: 22 ) and was the only patient who had revision surgery 3 years after initial surgery.

The mean values as well as the median values including the interquartile range (IQR) of the CT-scores and the olfactory assessment are listed in Table 1.

The analysis of the three different test methods showed a moderate positive correlation in the preoperative measurements between the VAS score and the Smell Diskettes $(0.72, p<0.001)$ and between the VAS score and the N-Butanol score $(0.81, \mathrm{p}<$ $0.001)$. After 3 months, the correlation was weak and did not reach the level of significance between VAS and Smell Diskettes $(0.19, p=0.21)$ and for the VAS and the N-Butanol $(0.28$, $p=0.07)$. After 5 years, the correlation was moderately positive again for VAS and Smell Diskettes $(0.56, \mathrm{p}=0.001)$ and for VAS and N-Butanol $(0.68, \mathrm{p}<0.001)$. 


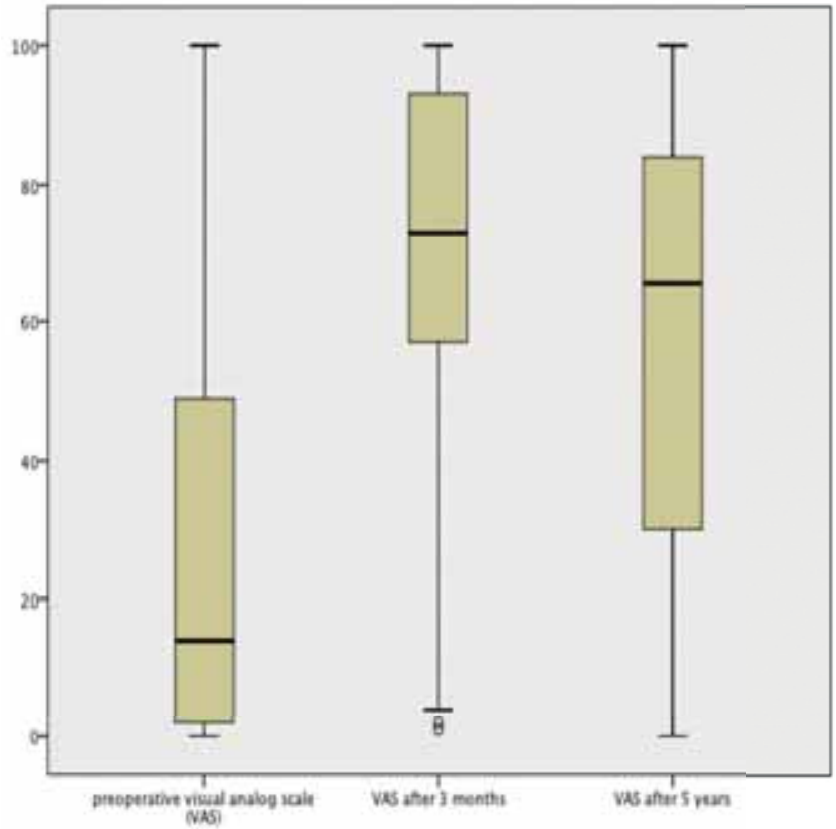

Figure 1. Visual analog scale rating of smell performance $(0=$ no sense of smell, $100=$ normal sense of smell) before surgery, after 3 months and 5 years. The improvement of the VAS values between preoperative and 3 months $(p<0.001)$ and 5 years $(p<0.001)$ were both significant. The tendency to lower values after 5 years compared with the values after 3 months did not reach significance $(p=0.037$; level of significance after Bonferroni-correction: <0.017).

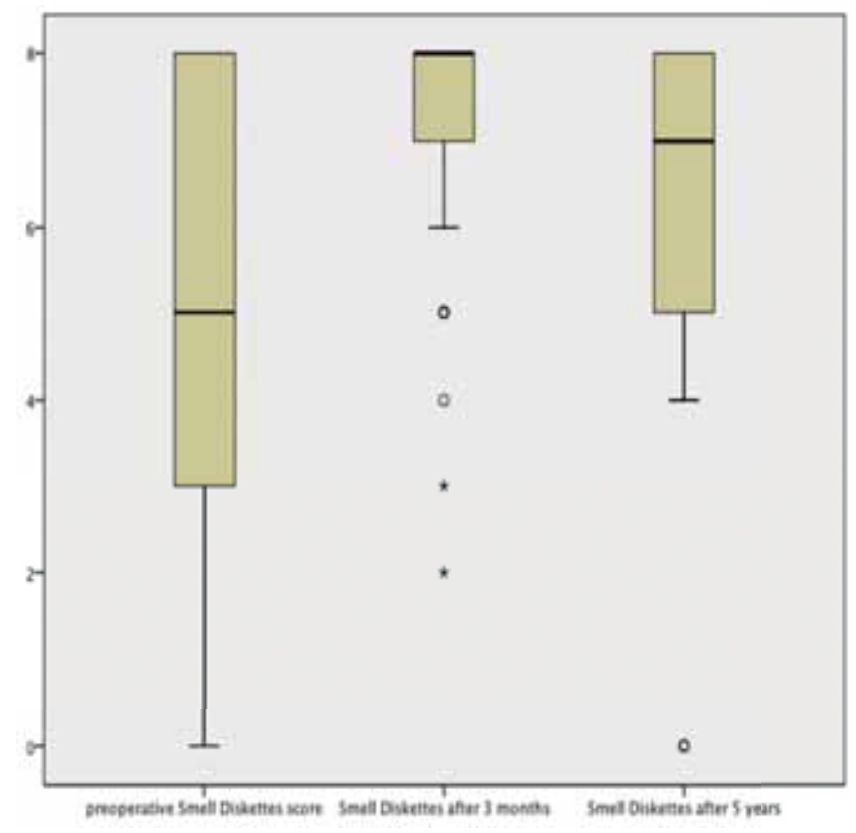

Figure 2. Smell Diskettes Test score $(0-6=$ anosmia or hyposmia, $7-8=$ normal) before surgery, after 3 months and after 5 years. The improvement of the mean score compared with the preoperative score was significant for 3 months $(p<0.001)$ but did not reach significance after 5 years $(p=0.063)$. The decrease of the score after 5 years compared with the score 3 months postoperative was significant $(p<0.001)$.
There was a moderate positive correlation between the preoperative Smell Diskettes and the $\mathrm{N}$-Butanol scores $(0.70, \mathrm{p}<0.001)$. After 3 months, this correlation was weaker $(0.48, p=0.004)$ and after 5 years it was stronger again $(0.65, p<0.001)$.

\section{Analyzing correlations between the preoperative CT-score} and olfaction scores, a negative correlation was found for all three tests preoperatively (VAS: - 0.62; Smell Diskettes: - 0.59; N-Butanol threshold: - 0.64). This means that a high preoperative CT-score showed a moderate correlation with lower olfactory performance. No correlation was found between the preoperative $\mathrm{CT}$ score and the olfaction scores after 3 months and after 5 years. However, a weak positive correlation was found between the CT-score and the degree of olfactory improvement after three months for all olfaction tests (VAS: 0.47; Smell Diskettes: 0.46; N-Butanol threshold: 0.42). This means that a high CT-score has a weak correlation with a better improvement of the olfactory performance after 3 months.

\section{Discussion}

One of the main symptoms of patients with chronic rhinosinusitis is the impaired sense of smell. The inflammatory changes of the nasal mucosa defining the chronic sinusitis lead to blockage of the olfactory cleft ('conductive' loss of smell). Furthermore, the inflammation of the olfactory mucosa itself may impair the olfactory function. It is well known that successful treatment of the underlying chronic rhinosinuisits may improve olfaction. If medical treatment alone is not sufficient to control the symptoms of the disease, endoscopic sinus surgery is indicated. The principle of endoscopic sinus surgery is to widen blocked ethmoidal drainage pathways and therefore restore drainage and ventilate the paranasal sinuses. An additional positive effect of opening the ethmoidal system is that topical steroids get better access to the diseased mucosa. Endoscopic sinus surgery is recognised as having the potential to improve olfaction but studies have not reported the long-term results ${ }^{(5-11)}$.

Comparing the three methods of olfactory assessment used in this study, it is known that subjective assessment with a visual analogue scale does not reflect the objective olfactory performance alone, but is confounded with other factors such as nasal breathing ${ }^{(16)}$. This is why preoperative assessment of olfaction should not rely on subjective methods alone but needs to be combined with objective smell tests ${ }^{(17)}$. In our patients, we found a moderate positive correlation between the VAS and the Smell Diskettes as well as with the N-Butanol threshold in the preoperative evaluation. This moderate correlation disappeared after 3 months. This finding may be explained by confounding factors like better nasal breathing, which may lead to a better subjective rating of olfaction. Another confounding factor may 


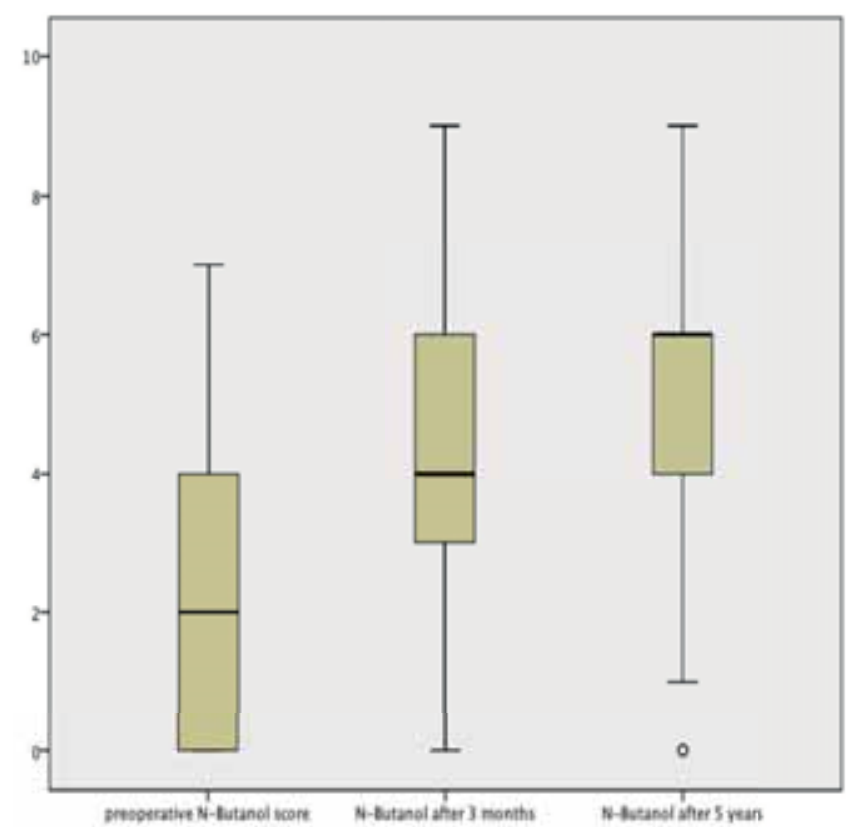

Figure 3. Threshold test of olfaction ( $\mathrm{N}$-Butanol) score $(0=$ threshold $>4 \mathrm{mg} / \mathrm{dl}, 9=$ threshold $=0.00061 \%$ ) before surgery, after 3 months and after 5 years. The improvement compared with the preoperative score was significant for 3 months $(p<0.001)$ and for 5 years $(p<$ $0.001)$. The tendency to a better score after 5 years compared with the score 3 months postoperative did not reach significance.

be a subjective expectation for improvement short time after the operation, which may lead to an over- or under rating of olfactory performance. After 5 years, the correlation between VAS and the Smell Diskettes score and the N-Butanol score was became moderately positive again. An explanation for this finding may be that the short time postoperative confounding factors disappeared and the subjective rating of olfaction reflected the objective olfactory performance in a more real way again.

Among the two objective methods used, it is known that the measurement of the olfactory threshold ( $\mathrm{N}$-butanol) is more precise in indicating the degree of the loss of smell than a screenig test (Smell Diskettes) ${ }^{(18)}$. Since screening tests are suprathreshold tests, the detection of small changes in the degree of olfactory performance cannot be expected. This may explain our finding, that the correlation between the Smell Diskettes und the N-Butanol scores was weaker after 3 months when the olfactory changes were most pronounced than after 5 years. However, a screening test was included in our evaluation because it is usually used in clinical practice because it is fast and cheap and gives important overall information about olfaction.

In our series of patients with endoscopic sinus surgery for chronic sinusitis, we found a high percentage of patients with subjective (visual analogue scale) and objective (Screening test with Smell Diskettes, N-Butanol threshold) improvement of their olfactory function not only three months postoperative but also after five years. The improvement in the mean scores was statistically significant for all test methods after 3 months and also after 5 years for the subjective assessment by VAS and for the sensitive N-Butanol threshold testing. Only the scores of the Smell Diskettes test did not reach a significant level of improvement at 5 years. This can be explained by the fact, that the Smell Diskettes test is less sensitive in measuring moderate changes of the olfactory performance. However, the percentage of normosmic patients as defined by the Smell Diskettes test improved from $38 \%$ to $75 \%$ after 3 months and also after 5 years it was still 56\%. This indicates that also in the Smell Diskettes test the improvement of olfaction is reflected, even if it reaches not a statistic significant level.

Overall, the improvement after three months was better than after five years, which reflects the tendency of recurrence of the underlying disease process. Another factor for the decrease in olfactory performance after 5 years may be a fading compliance for the treatment with topical medication. Although the patients were followed up regularly and topical medication was recommended based upon disease activity, some patients stopped this topical therapy because they were satisfied with their symptoms. The decrease in olfactory performance did not reach statistical significance in the subjective VAS measurement and in the N-Butanol threshold but was significant with the Smell Diskettes test. This difference may be explained by the fact, that methods used to evaluate olfaction do not have the same detection level and may also be confounded by other factors such as nasal breathing (VAS).

In summary, the high percentage of patients with improvement in their sense of smell five years after surgery measured by subjective visual analogue scale (79\%) and by objective testing (Smell Diskettes $=53 \%$; N-Butanol threshold $=85 \%$ ) indicate that endoscopic sinus surgery leads to a long term improvement of olfaction compared with the situation before the operation with medical treatment alone.

One technical aspect of surgery in our series was the focus on a good 'ventilation' of the olfactory cleft by gentle 'lateralisation' of the middle turbinate at the end of the procedure. It is not clear, whether this surgical concept leads to a better long-term result regarding the sense of smell. However, there were no complications due to a blocked frontoethmoidal or maxilloethmoidal drainage pathway in our series and the good overall long-term results indicate that a good 'ventilation' of the olfactory cleft may be one factor of a stable long-term result. Another finding in our analysis was that in $9 \%$ of the patients analyzed by the most 
precise N-Butanol threshold stayed unchanged after 3 months and 5 years. Furthermore, a number of patients ( $9 \%$ after 3 months and $6 \%$ after 5 years) even had a deterioration of their threshold level. This finding is consistent with other reports, for example Pade et al., found a decrease of olfactory function in $9 \%$ of patients 4 months after endoscopic sinus surgery ${ }^{(19)}$. The reason for this deterioration after surgery is unclear; a probable mechanism is the ongoing inflammatory process of the underlying disease, which may lead to a further loss of olfaction and the fading compliance in medical treatment as mentioned above. However, a negative impact of the surgical procedure itself may not be ruled out. The fact that $15 \%$ of the patients have no long term improvement or are even confronted with a further deterioration of their sense of smell after surgery should be mentioned in the preoperative counselling of the patients.

High preoperative CT-scores showed a moderate correlation with a lower olfactory performance. This means that patients belonging to the subgroup with chronic rhinosinusitis with polyps had a tendency to have a more severe loss of smell compared with patients without polyps. This finding is consisting with other reports and indicates that a high CT-score is found in patients with blockage of the olfactory cleft by polyps causing severe hyposmia ${ }^{(8-10,20)}$. Another finding consisting with previous reports was that there was some positive correlation between a high preoperative CT-score and the degree of improvement of olfaction after 3 months ${ }^{(8,19)}$. This correlation was weak and it it stands in contrast to other reports which found that the degree of chronic rhinosinusitis and the coexistence of nasal polyps had no prognostic impact on the improvement of the sense of smell 6 months after endoscopic sinus surgery (21) or that severe nasal polyposis even correlated with impaired olfactory improvement ${ }^{(20,22)}$. This divergence in the literature regarding the prognostic factors may be explained by various confounding factors influencing olfactory outcome such as comorbidity (for example asthma), the extent of surgery performed on the individual patient, or the type and extent of medical treatment in the postoperative period. Further studies are needed to determine the prognostic role of these confounding factors on long-term olfactory improvement.

\section{Conclusion}

Endoscopic sinus surgery is a well-established treatment in patients with chronic rhinosinusitis, who do not sufficiently respond to medical treatment. It is widely recognized that endoscopic sinus surgery improves the sense of smell along with other nasal symptoms. However, most studies only analyse olfactory function one year or less after surgery and little is known about the long-term result of olfaction. In our series of 45 patients, we could confirm a statistically significant subjective and objective improvement of the olfactory function after 3 months. Furthermore we could follow up 34 of these 45 patients (76\%) after five years and found a persistent improvement in their sense of smell, although it was not as high as it was after three months. The high percentage of patients with improvement of olfaction five years after surgery measured by subjective visual analogue scale (79\%) and by objective testing (Smell Diskettes $=53 \%$; N-Butanol threshold $=85 \%$ ) indicates that endoscopic sinus surgery in conjunction with topical medical treatment leads to a long term improvement of the sense of smell.

On the other hand it was found that - measured by the sensitive $\mathrm{N}$-Butanol threshold - up to $15 \%$ of the patients did not have an improvement or even had a further deterioration in their olfaction 5 years after endoscopic sinus surgery. This fact has to be considered in the counselling of the patients before surgery too.

\section{Acknowledgement}

The authors thank Professor Dr. Burkhardt Seifert, Biostatistics Unit ISPM, University of Zurich, Hirschengraben 84, CH-8001 Zurich, Switzerland, for performing the biostatistical analysis.

\section{References}

1. Damm M, Quante G, Junghuelsing $M$, Stennert E. Impact on functional endoscopic sinus surgery on symptoms and quality of life in chronic rhinosinusitis. Laryngoscope. 2002; 112: 310-315.

2. Fokkens WJ, Lund VJ, Mullol J, et al. European Position Paper on Nasal Polyps. Rhinology. 2007; 45, suppl 20: 1-139.

3. Delank KW, Stoll W. Olfactory function after functional endoscopic sinus surgery for chronic sinusitis. Rhinology. 1998; 36: 15-19.

4. Blomquist EH, Lundblad L, Anggard A Haraldsson PO, Stjarne P. A randomized controlled study evaluating medical treatment versus surgical treatment in addition to medical treatment of nasal polyposis. J Allergy Clin Immunol. 2001; 107: 224-228.

5. Rowe-Jones JM, Mackay IS. A prospectice study of olfaction following endoscopic sinus surgery with adjuvant medical treatment. Clin Otolaryngol Allied Sci. 1997; 22: 377-391.

6. Shin SH, Park JY, Sohn JH. Clinical value of olfactory function after endoscopic sinus surgery: a short-term result. Am J Rhinol. 1999; 13: 63-66.

7. Jankowski R, Bodino C. Olfaction in patients with nasal polyposis: effects of systemic steroids and radical ethmoidectomy with middle turbinate resection (nasalisation). Rhinology. 2003; 41: 220230.

8. Perry BF, Kountakis SE. Subjective improvement of olfactory function after endoscopic sinus surgery for chronic rhinosinusitis. Am J Otolaryngol. 2003; 24: 366-369

9. Jiang RS, Lu FJ, Liang KL, Shiao JY, Su MC, $\mathrm{H} \sin \mathrm{CH}$, Chen WK. Olfactory function in patients with chronic rhinosinusitis before and after functional endoscopic sinus surgery. Am J Rhinol. 2008; 22: 445-448.

10. Litvack JR, Mace J, Smith TL. Does olfactory 
function improve after endoscopic sinus surgery? Otolaryngol Head Neck Surg. 2009; 140: 312-319.

11. Olsson P, Stjarne P. Endoscopic sinus surgery improves olfaction in nasal polyposis, a multi-center study. Rhinology. 2010; 48: 150-155.

12. Lund VJ, Mackay IS. Staging in rhinosinusitis. Rhinology. 1993; 31: 183-184.

13. Lund VJ, Kennedy DW. Staging for rhinosinusitis. Otolaryngology - Head and Neck Surgery. 1997; 117: S35-S40.

14. Briner HR, Simmen D. Smell Diskettes as Screening Test of Olfaction. Rhinology. 1999; 37: 145-148.

15. Cain WS, Goodspeed RB, Gent JF, Leonard G. Evaluation of olfactory dysfunction in the Connecticut Chemosensory Clinical Research Center. Laryngoscope. 1998; 98: 83-88.

16. Landis BN, Hummel T, Hugentobler M, Giger R, Lacroix JS. Ratings of overall olfactory function. Chem Senses. 2003; 28: 691-694.

17. Philpott CM, Rimal D, Tassone P, Prinsley PR, Premachandra DJ. A study of olfactory testing in patients with rhinological pathology in the ENT clinic. Rhinology. 2008; 46: 34-39.

18. Lotsch J, Reichmann H, Hummel T. Different odor tests contribute differently to the evaluation of olfactory loss. Chem Senses. 2008; 33: 17-21.

19. Pade J, Hummel T. Olfactory function following nasal surgery. Laryngoscope. 2008; 118: 1260-1264

20. Young J, Frenkiel S, Tewfik MA, Mouadeb DA Long-term outcome analysis of endoscopic sinus surgery for chronic rhinosinusits. Am J Rhinol. 2007; 21: 743-747

21. Jiang RS, Su MC, Liang KL, et al. Preoperative prognostic factors for olfactory change after functional endoscopic sinus surgery. Am J Rhinol Allergy. 2009; 23: 64-70.

22. Katotomichelakis M, Riga M, Davris S, Tripsianis G, Simopoulou M, Nikolettos N
Simopoulos K, Danielides V. Allergic rhinitis and aspirin-exacerbated respiratory disease as predictors of the olfactory outcome after endoscopic sinus surgery. Am J Rhinol Allergy. 2009; 23: 348-353.

Hans Rudolf Briner, MD

ORL-Zentrum

Klinik Hirslanden

Witellikerstrasse 40

$\mathrm{CH}-8032$ Zürich

Switzerland

Tel: +41-44-387 2800

Fax: +41-44-387 2802

E-mail: briner@orl-zentrum.com

UCL EAR INSTITUTE

GRAYS INN ROAD COURSES

盖

An Endoscopic Approach

to Rhinosinusitis

3-6 October 2012

Faculty:

V J Lund, D Roberts, LBadia,

T Beale \& H Kariyawasam

Guest Lecturer: Professor Heinz Stammberger

The successful and well established course covers all aspects of rhinosinusitis, advanced approaches to the frontal sinus including balloon sinuplasty, extended endoscopic surgery of the skull base and orbit and the use of computer assisted surgery from a very experienced faculty using:

- Hands-on anatomy dissection

- Anatomical demos

- $\quad$ Live operating

$£ 845$ with

dissection*

$£ 475$ without

dissection

mited spaces availa

Course STOR2 NeilMed

sponsors STOR2 SINUS RINSE

Visit: ww w.ucl.ac.uk/ear/courses

Contact: +44 (0)20 $76798909 / 8964$

email: a.kelly@ucl.ac.uk for dissection)

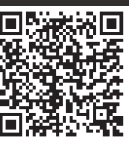

